\title{
IMPROVED CLOSED ORBIT MEASUREMENT SYSTEM FOR PLS
}

\author{
J. Y. Huang*, S. J. Park, W. H. Hwang, D. T. Kim, and S. H. Nam, \\ Pohang Accelerator Laboratory, San 31, Hyoja-dong, Pohang 790-784, Korea
}

\begin{abstract}
With the installation of the first insertion device U7 and more and more sophisticated experiments in the Pohang Light Source (PLS), the synchrotron radiation users now demand more accurate measurement and control of the closed orbit. As the essential part of the accurate orbit measurement and feedback, all the BPM electronics and data acquisition system have been upgraded. In the new BPM system, we adopted a commercial BPM processor, which has better than $5 \mu \mathrm{m}$ accuracy over $65 \mathrm{~dB}$ dynamic range of input signal. The data acquisition board is made in-house utilizing a fast ADC and an on-board microprocessor. New BPMs and various machine diagnostics on the orbit drift with the aid of new BPMs will be described in this paper.
\end{abstract}

\section{INTRODUCTION}

PLS[1] is a third generation 2.0-2.5 GeV electron synchrotron light source dedicated to users since 1995 . Until 1999, the PLS storage ring had been run at $2.0 \mathrm{GeV}$ (phase-I operation) with only bending magnet beamlines and there had been little demand for the precise closed orbit control from the beamline users. PLS is now in the advanced(phase-II) operation mode with the first insertion device(ID) $\mathrm{U} 7$ at higher beam energy $2.5 \mathrm{GeV}$ with $180 \mathrm{~mA}$ injection current from the year 2000 . Although the previous in-house made BPMs [2] with $20 \mu \mathrm{m}$ resolution played very important role during the commissioning and the phase-I operation, more accurate measurement and feedback of the closed orbit with few micrometer resolution is required for the phase-II operation. Considering these requirements, all the BPM electronics have been replaced in 1999 with commercial (Bergoz) BPM processors [3], which have better than $5 \mu \mathrm{m}$ accuracy over $65 \mathrm{~dB}$ dynamic range of input signal. The analog to digital conversion(ADC) and data acquisition(DAQ) boards are made in-house utilizing onboard microprocessors for the local intellegence. Each DAQ board has separate RS232 and RS485 interfaces for the offline access to the local database. Thus all the BPM boards can be linked serially through RS485 for offline applications of BPM data, such as beam-based alignment(BBA) system [4].

Various beam diagnostics and investigations on the source of the measurement errors are in progress with new closed orbit measurement system. Most important issue is the orbit drift caused by the temperature change of the vacuum chamber, magnet and air in the storage ring tunnel. Preliminary experiment showed that the orbit

*huang@postech.ac.kr stability was significantly improved by the temperature stabilization of these components [5]. Other BPM position offset errors come from the difference of pickup gains as well as the phase mismatching between signal cables, which can be compensated only by the BBA technique.

\section{NEW BPM SYSTEM}

In the 12-cell, 280m PLS storage ring, there are 9 BPMs in each cell(total 108 BPMs) for the measurement and control of the closed orbit. In addition, 2 IDBPMs are installed on each straight section chamber for the steering of ID orbit. Among various analog signal outputs from a BPM board, only the beam position( $x$ and $y$ ) and AGC signals are digitized for regular operational database. There are two 24-channel $100 \mathrm{kHz} 12$-bit ADC boards in each lattice cell. Resolution of position reading is improved to $4 \mu \mathrm{m}$ with 128 times averaging of the raw BPM data in the microprocessor. By averaging, it gives the maximum $33 \mathrm{~Hz}$ reading-rate for each position. Presently, the ultimate resolution is limited to $4 \mu \mathrm{m}$ by 12bit truncation of the averaged data in the 12bit microprocessor. To further improve the resolution, the 12 bit microprocessor will be replaced with a 16bit one or do floating point computing in the local VME control computer. Each ADC board has RS232 and RS485 interfaces for local access to the BPM data providing much convenience for the offline beam physics experiments. Fig. 1 shows a schematic circuit of the new BPMs.

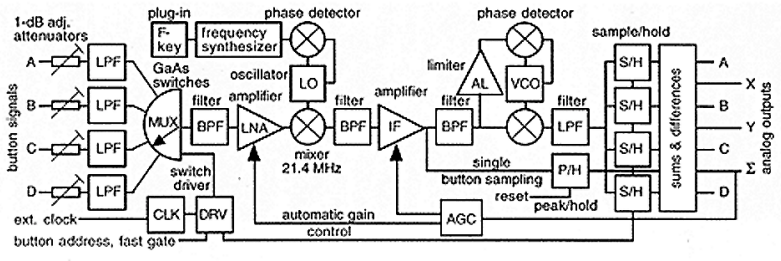

Fig.1: Schematic circuit diagram of the Bergoz BPM. See Ref.[3] for more details.

\section{PERFORMANCES}

\subsection{Resolution and Long-Term Stability}

Performance test of the new BPM had been done in the test lab by simulating the beam with a rf antenna [5]. Resolution is measured by moving the antenna with a stepping motor. At the same time, antenna position is measured by a laser position detection sensor(LDS) which has $0.3 \mu \mathrm{m}$ resolution. In Fig. 2 , it shows clearly that the BPM has less than $1 \mu \mathrm{m}$ resolution. Long-term stability 
was also checked by monitoring the position variation of the antenna caused by the change of the room temperature in the test lab. As the temperature rises, antenna wire expands slightly leading to the transverse displacement of a few micrometers. In Fig. 3, the antenna movement measured by LDS and the BPM signal agrees within the resolution $(<5 \mu \mathrm{m})$ of the BPM.

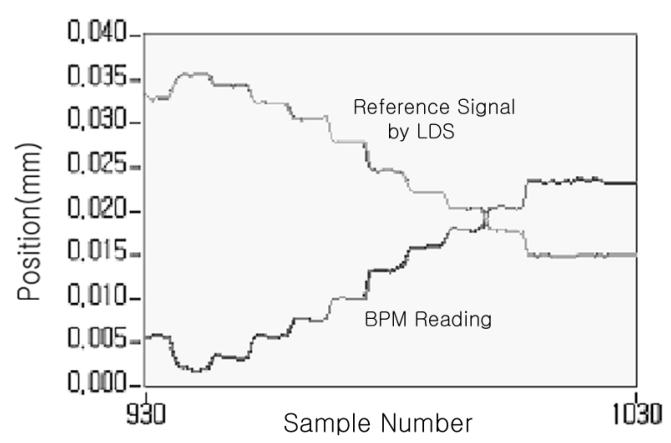

Fig.2: Resolution of the BPM measured with precision laser detection system. BPM agrees with LDS within $1 \mu \mathrm{m}$.

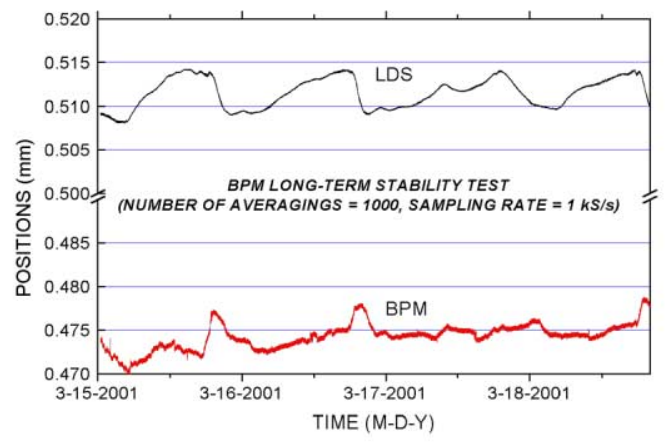

Fig. 3: Drift of the BPM measured for 90 hours.

\subsection{Offsets}

Because the BPM pickup modules are installed on a $10 \mathrm{~m}$ long, single-piece vacuum chamber, BPM offsets caused by differences in pickup gains could not be measured in the test lab. Instead, BBA technique will be used for the calibration of all kind of BPM offsets in the future. Phase mismatching of the BPM signal cable gives another error in position measurement. Since the pickup signal is reflected between the input port of the BPM electronics - such as low pass filter, band pass filter or just rf switches - and the pickup button, the reflected signal adds up to the beam signal at the input port. Thus the input signal amplitude depends on the phase difference between the beam signal and the reflected signal. Suppose the input signal as $\mathrm{x}_{0}(\mathrm{t})=\mathrm{x}_{0} \sin \omega \mathrm{t}$, and reflected signal as $\mathrm{x}_{1}(\mathrm{t})=\mathrm{Ax_{0 }} \sin (\omega \mathrm{t}+\varphi)$. Then the amplitude depends on the cable length as $\mathrm{x}_{0}\left[(1+\mathrm{A} \cos \varphi)^{2}+(\mathrm{A} \sin \varphi)^{2}\right]^{1 / 2}$. The phase difference between two signals is $\varphi=2 \pi(2 l) / \lambda$, where $l$ is cable length and $\lambda$ is the wavelength of beam signal. The maximum position error can be $1 \mathrm{~mm}$ (peak-topeak). By using $6 \mathrm{~dB}$ attenuators at all input ports, the error can be reduced to less than $100 \mu \mathrm{m}$ as shown in Fig. 4. The final calibration of the BPM offsets is still subject to the in-situ BBA technique [4]. Before BBA, we estimated all the pickup gains by measuring the cross coupling between pickup buttons and phase factors for all signal cables as shown in Fig. 5. The offset values are used as a look-up table in the computer to correct BPM offsets.

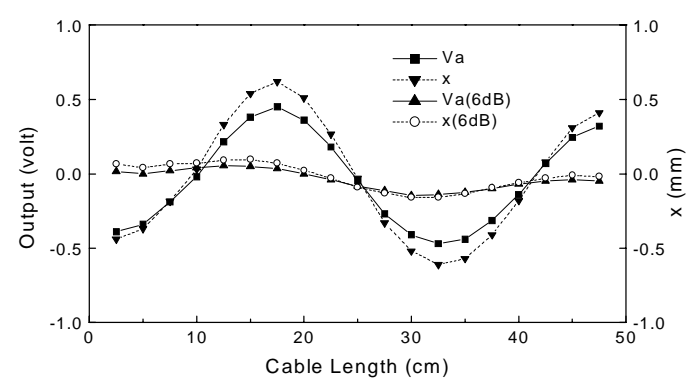

Fig.4: Phase effect on the length of the signal cable a. Peak-to-peak position error by phase mismatch can be 1 $\mathrm{mm}$ unless $6 \mathrm{~dB}$ attenuators are used. Va: channel-a signal(volt), $\mathrm{x}$ : position-x (mm)

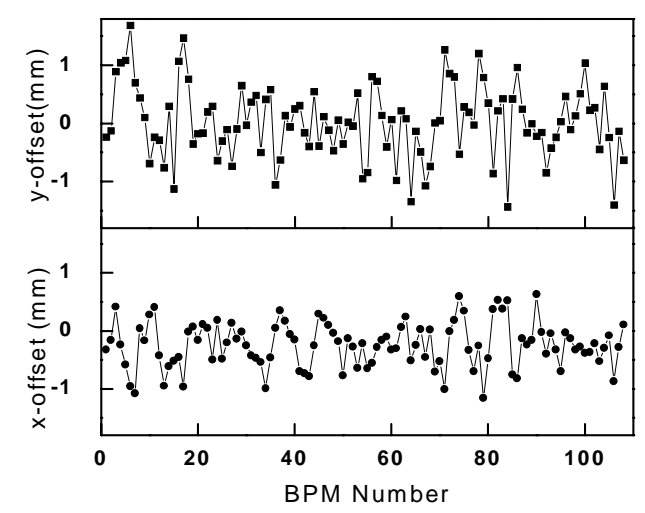

Fig. 5: BPM offsets measured in PLS. Phase mismatch between signal cables, pickup gains and cable losses are all included in the offset values.

\subsection{Connectors}

A pickup button connector is a $40 \mathrm{~mm}$ long, 50ohm matched air-dielectric SMA connector without a dielectric bushing to support the center conductor. Thus the mating force of the center conductor may become loose when it is handled without care. Indeed, some conductors have become loose making bad rf contacts. Each connector is checked by time domain reflectometric(TDR) measurements and corrected appropriately. Typical TDR signals for a loose connector before and after fixing of the mating spring are shown in Fig. 6. 


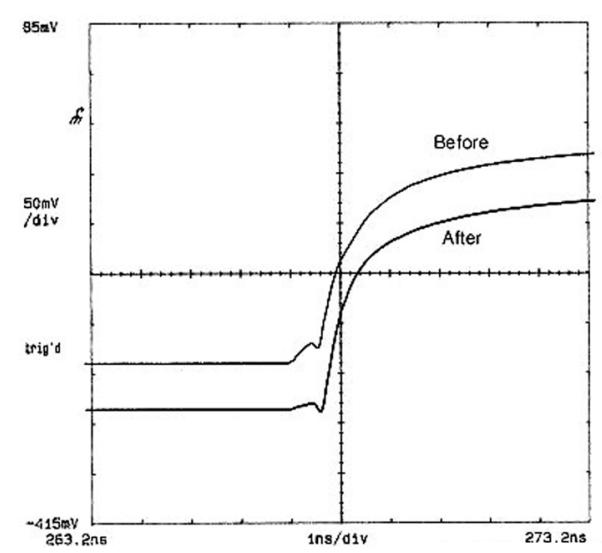

Fig. 6: TDR signal before and after the correction of the loose contact at the BPM connector.

\section{ISSUES IN THE ORBIT MEASUREMENT}

\subsection{Thermal Effects on the BPMs on the Vacuum Chamber}

Another important thermal problem is an error of beam position measurement from the BPMs installed on a $10 \mathrm{~m}$ long single-piece vacuum chamber. Chamber distortion by the temperature change gives an error to BPM. In a preliminary test of cooling a $10 \mathrm{~m}$ long vacuum chamber, the BPM reading error reduced significantly indicating that all the chambers should be cooled in the future.

\subsection{Thermal Effect on the Orbit Measurement}

Long-term orbit measurements show also slow drift of the beam position up to $+/-150 \mu \mathrm{m}$ as shown in Fig. 7 . With careful investigations, we find that there is a strong correlation between the orbit drift and the temperature changes of the magnet and air in the storage ring tunnel. Major source of the temperature change comes from the large change of the magnet currents during the regular beam injection. With a novel de-ramping and ramping process for beam injection, thermal shock on magnets reduced significantly [5,6]. Further studies on various thermal effects are in progress to stabilize temperature drifts in the storage ring components.

\subsection{Effect of Storage Ring Deformation}

The settlement of the storage ring tunnel continues at about $3.0 \mathrm{~mm}$ (hill to valley) per year in PLS [6]. The total deformation has reached presently $19 \mathrm{~mm}$ compared to the reference elevation established in June 1993. The amount of the orbit drift with the ground motion would be $200 \mu \mathrm{m}$ in a ten-day user run period. Since the accumulated deformation still increases without slowdown, we decided to realign all storage ring component toward the design orbit step by step in three years. Nevertheless, the relatively large position change by the ground motion should be cured by a realtime orbit feedback system in the future.

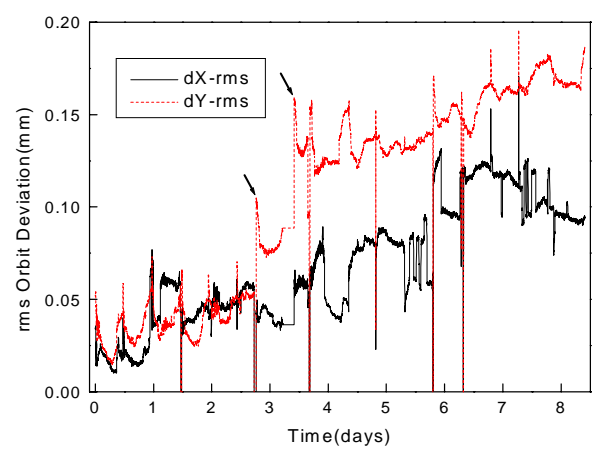

Fig. 7. RMS orbit deviation with operation time. Arrows indicate large increase of rms values by the degaussing of magnets by unexpected beam dumps.

\section{FUTURE PLANS}

Beam based alignment of the BPMs and beam optics is the most important project ongoing in PLS for the accurate and stable orbit measurement [7]. To stabilize the closed orbit, we still need to investigate more on the various thermal effects on the orbit drift. For this purpose, a task force team has been assembled for the extensive studies. Also we are planning a new diagnostic station equipped with a photon beam position monitor(PBPM) and a photon beam intensity monitor(PBIM) with thermal and mechanical isolation. With this reference monitors, we will be able to investigate on the source of the orbit measurement errors more effectively.

\section{REFERENCES}

[1] PLS Design Report (revised ed.), Pohang Accelerator Laboratory, 1992.

[2] J. Y. Huang, et. al., "PLS Beam Position Monitoring System," EPAC'92 Proceedings, pp1115-1117, (1993).

[3] Bergoz BPM, visit http://www.bergoz.com/.

[4] K. H. Kim, J. Y. Huang, and I. S. Ko, "The BBA Technique for the Measurement of BPM Offsets and Beam Offsets from Quadrupole Magnets in PLS", Jap. J. Appl. Phys. 38, 6926(1999). See also Ref. [7].

[5] S. J. Park, J. Y. Huang, W. H. Hwang, and S. H. Nam, "Improvement of Orbit Stability Using PLS Beam Position Monitoring System," submitted to KAPRA Conference, Cheju, Korea, July 2001.

[6] J. Y. Huang, et. al., "The Operational Status of Pohang Light Source," in this Proceedings.

[7] J. Choi, private communication. mailto:jchoi@postech.ac.kr. 